\title{
A Study of Topologically Society and Culture space on Taiwanese Settlement
}

\author{
Chi-Hua, Li \\ Assistant Professor, Department and Graduate School of Interior Design, National Taichung University of Science and \\ Technology, Taiwan. \\ lich@nutc.edu.tw
}

\begin{abstract}
Most of the traditional settlement studies are based on text description of the social or cultural perspectives or drawing records of the spatial form of the settlements. It may be difficult to further compare the social and cultural space of a number of settlements, or to discuss the spatial structure of different settlements. This study proposes a topological analysis method of mathematical theory to understand the inherent spatial logic of settlements in Taiwan. Qualitative research methods such as field investigation are adopted to obtain the settlement life information and define the space for abstraction. Starting from the geometric concepts in the topological space, this stud explores the geometric spatial relationships and calculates the values of various points to learn the characteristics of each space for the interpretation of the space from the concept of saturation and depth. Such a research method describes the social and cultural significance of different spaces from another angle, and compares the differences in the settlement spatial structure by values.
\end{abstract}

Index Terms - topology, settlement, culture form, Saturation

\section{Introduction}

Settlement is the mark of human living space. It not only reflects the various economic, political, cultural behavior patterns of contemporary human beings, but also accumulates the life patterns in the history of the space. Historical events not only refer to the time of human behavior occurrence, but the development process of human activities. The settlement development process is recorded in the traditional residence, settlement and urban space through regional characteristics and the impact of different eras. It is also reflected by the use behavior, spatial patterns and living culture. When the residential form enters the age of industrial production, the organic evolution of neighborhood atmosphere, a sense of selfevidence and the place of a traditional living space has been difficult for the residents in modern living space to perceive.

In addition to descriptions by text and language, spatial society- oriented research on settlement has unspeakable difficulties regarding the description of the actual operations of the design. Furthermore, many lifestyles in settlements have gradually disappeared, and it is not easy for researchers to imagine how the past people lived from the fragments in space form.

In this study, in a logical language, we describe sociocultural and spatial relationships constructed by everyday life in settlements by using three different maps: satellite images, topographic maps and maps of daily life activities. These three kinds of maps express different settlement knowledge. By attempting to expand the research scope to present the spatial relationship, the relationship between space and life, and the relationship between society and culture, it is expected to specifically present the relatively obscure relationship between people and space to explore human, space, life and social culture interactions in settlements. Through logical language and the understanding and perception of space, this paper constructs logical descriptions and operating procedures for the study of the logic of traditional space construction through a variety of collective memory of traditional Taiwanese settlement and the space model formed by the interactions of the individuals, groups and space.

\section{Research Method}

In terms of the academic community, numerous theories have been applied in the spatial computing designs [5].[7].[8]. Among the studies similar to topology analysis, Hillier \& Hanson (1984), proposed in the book entitled The social logic of space [1] the "space syntax" research method, to explore correspondence between the real architectural and urban space and functional activities. Such links of abstracted spatial relationships similar to the bubble diagrams of construction plan should not be unfamiliar for designers.

Based on topology theory, space syntax is helpful for the preliminary study of a space. However, it cannot clearly define settlement space in Taiwan. life practice and spatial form of the traditional settlement, by sound transmission, active links, behavioural differences, can be defined as the space factors. Accordingly, Li (2010) [4].proposed the principle-defined space by abandoning the concept of coupler diagram, but regarding space as nodes and the relationships between spaces as links. This method can fully consider the dynamics and activities of the settlements. Moreover, after the computation of the distance of each route from a node to any other node in the space, it is found that the concept of the shortest route and the value are not the only indicators; instead, the concepts of saturation and depth are the indicators in the discussion of the inner connection of space and life in Taiwan's traditional settlements.

Saturation: For a node, the depth value may increase when step increases. A node is saturated when its maximum depth value is reached. In other words, the depth value will not change further, even though one can step further. 
Root node: Every node in a graph can be regarded as a root node. Thus, a particular minimum spanning tree can be constructed with respect to it.

Layer: the root node is labeled as in layer 0 , and every unlabeled node connected to a node in layer $i$ is labeled as in layer $\mathrm{i}+1$.

Stepwise Depth $D_{s}^{j}$ (the number of nodes within s layers (steps) with respect to node $\mathrm{j}$ ): Let node $\mathrm{j}$ be the root with $\mathrm{k}$ layers, and there are $\mathrm{n}_{\mathrm{i}}$ nodes at layer $\mathrm{i}(\leqq \mathrm{k})$, the number of nodes within $\mathrm{s}(\leqq \mathrm{k})$ layers with respect to node $\mathrm{j}$ is $\mathrm{D}_{\mathrm{s}}^{\mathrm{j}}=\sum_{i=1}^{s}\left(i \times n_{i}\right)$.

Please be noted that the concept of $\mathrm{D}_{\mathrm{s}}^{\mathrm{j}}$ is realized as 'depth' by Hillier \& Hanson (1984) from the viewpoint of urban space analysts, and movements across layers is regards as steps. Thus, one-step depth (D1), two-step depth (D2), three-step depth (D3), and total depth mentioned in space syntax are respectively corresponding to $D_{1}{ }^{j}, D_{2}{ }^{j}, D_{3}{ }^{j}$ and $D_{k}{ }^{j}$.

After the numerical computation, the meanings of the following three indicators will be explored:

1) Centrality can be derived from saturation.

2) The first $20 \%$ saturated nodes are keys of partitions.

3) Inaccessibility can be indicated by the later saturated nodes.

$\mathrm{Li}$ (2009) [3]., the initial deduction of the links is made between spatial topology and sociality. This study continues the research theory to verify whether the research theory is applicable to the case in central Taiwan.

\section{Case Study on Traditional Settlements in Taiwan}

In this study, Shui-Duei settlement founded in 1710 to the present day in the central Taiwan is selected as the case for study. Shui-Duei settlement is a family-based settlement. Starting from a traditional courtyard in 1710 , with gradually increasing population, the settlement has become a complex of buildings including three courtyards with five guarding walls and other ancillary buildings. The development reached a stable state in 1970s. The settlement has its own festival and religious culture, as well as active neighborhood interactions. Currently, the application of classifying Shui-Duei settlement as a living settlement is waiting for the approval of the Taichung City Government. The area is as shown in Figure 1.

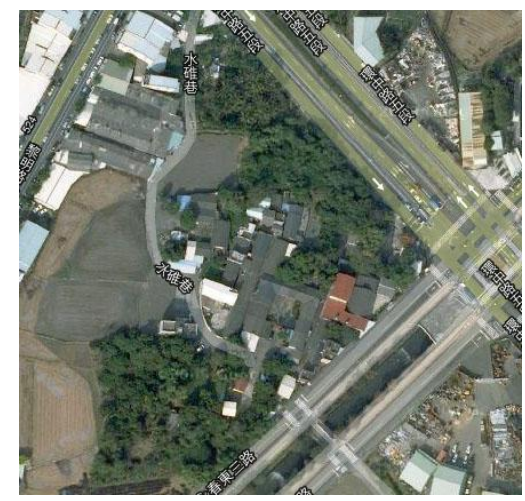

Fig. 1 Satellite photo of Shui-Duei settlement (Google map)

\section{Spatial Topology}

After the field investigation, recording and interviews of the settlement, With the behaviors of daily life as the focus of the field investigation, this paper defines the space and identify the nodes in the space. According to the spatial characteristics, the space was divided into two types, public space and private space. The public space includes plazas, roads, large trees, veranda, temples and other places that are freely accessible. Private space includes private residence, private industry, and other places that are not accessible without permission. In this study, all of the spaces are nodes. Nodes in capital letters, such as node $\mathrm{A}$, nodeB, nodeC, and nodeD denote the public space; nodes in Arabic numerals, such as node 1, node 2, node 3, and node 4 denote the private space. All the space in the settlements is encoded and given a set of codes. Furthermore, the relationships of the nodes in the settlement are identified. The nodes are connected and represented by link, which indicates the accessible relationship between nodes. After linking up the nodes, the relationships are converted into the topological structure as shown in Figure 2. In the topological relationship diagram, the layer relationship of the space can be clearly seen. Finally, after computing the depth and number of steps of each individual node, the results of the public space are discussed and analyzed.

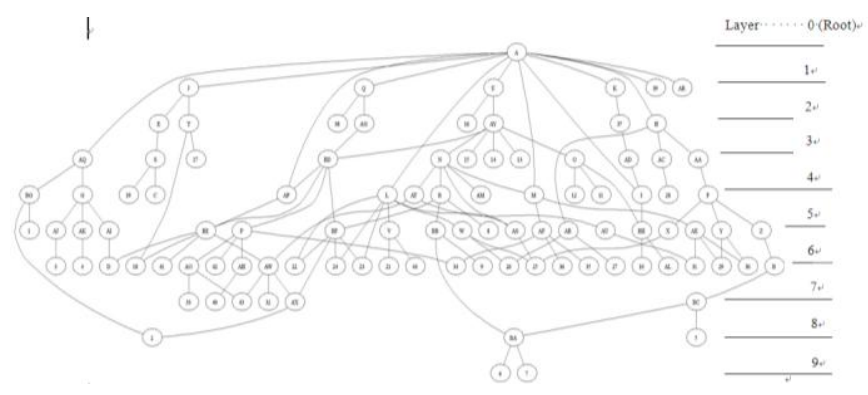

Fig.2 Spatial topology diagram of Shui-Duei settlement

The conversion of topological space demonstrates the space-level problems. As shown in Figure 2, the number of layers in the topological structure is up to 9 . In the actual experience of space, the spatial meanings become complexity and varied. The residents are familiar with each other; strangers will be identified immediately once entering the settlement. In a preliminary observation, the relationship between the spatial culture and spatial structure suggests that the settlements have good depths in spatial layers due to the long history and good neighbourhood interactions. Compared to the four layers of the Taipei Minsheng Community, which was developed by the government after World War II, the two layers of the student dormitory building of National Taiwan University is rather shallow and lacks spatial connectivity. Therefore, from the perspective of resident interaction and sense of community, it is more easily for the deep structure to generate self-evident and better spatial neighbourhood relations. The two types of traditional settlements explored in 
Li \& Lin(2010) [4]. have deep structures, and the Shui-Duei settlement also has a deep structure.

According to the computation results of Stepwise depth $D_{s}^{j}$ as shown in Figure 2, the depth and saturation of the spatial nodes in the Shui-Duei settlement are obtained. The first saturated with 7 steps include node $\mathrm{A}$, node $\mathrm{H}$, nodeM, node $\mathrm{N}$, nodeO, nodeU, nodeAA, nodeAE, nodeAF, nodeAG, nodeAM, nodeAS, nodeAW, nodeAY, nodeBD, nodeBF, nodeBH; the lowest $20 \%$ includes nodes nodeA, nodeU, nodeL, nodeM, nodeJ, nodeAQ, nodeH, nodeAP, nodeQ, nodeAY, nodeBD as shown in Figure3.

The latest saturated with 12 steps is node B, and its value is the highest (733) Nodes that are saturated with 11steps include nodes Z, BA, Y. Nodes that are saturated with 10 steps include nodes $\mathrm{X}$, nodeAS, node $\mathrm{AK}$, nodeBB, nodeAT, nodeBC. These nodes are $20 \%$ of the highest value as shown in Figure4.

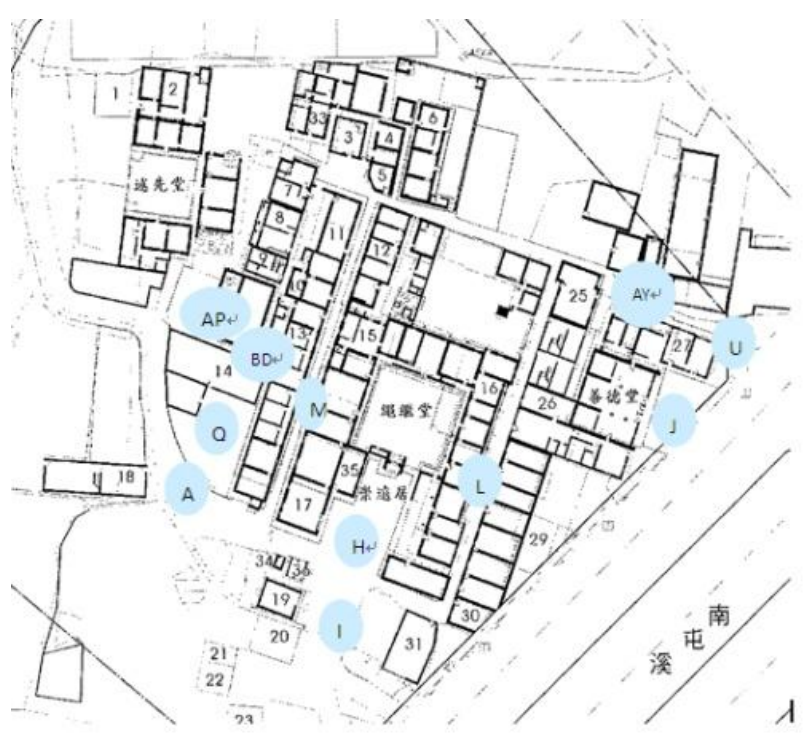

Fig. 3 The first $20 \%$ saturated nodes

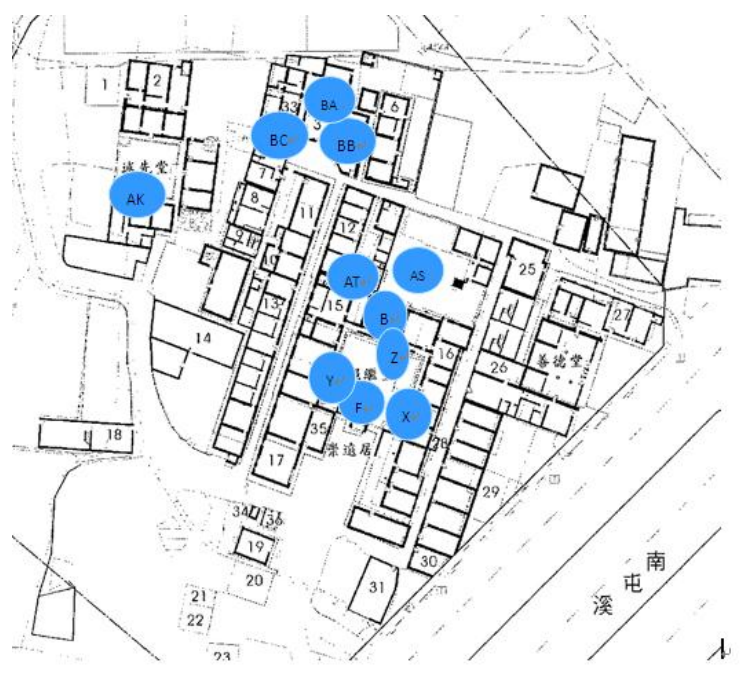

Fig. 4 The latest $20 \%$ saturated nodes

\section{Conclusion}

Topology analysis is one of the major topics in the discussion of spatial features. This study applied the numerical concept in study, and used the basic topological pattern to discuss the spatial structural relationships of the Shui-Duei settlement.

(1) Regarding the depth calculation results, in addition to using the values to compare the center/edge concepts, the saturation concept of statistics is used as the basis for the assessment of the spatial elements. The element that is saturated in the first place with lowest value is the center of the overall space. The element that is saturated in the last place with highest value is the edge of the overall space. Moreover, settlement center is a space of high accessibility with stronger public nature. Conversely, the settlement edge is a space of low accessibility with stronger privacy. The findings are as follows:

"Center / periphery" and the "public / private": the node at the center of the settlement with minimum value and the first saturated (7steps) is nodeA(296), which is the main access to the settlement and the main connection road with the parts of the settlement space. Therefore, it is the center of daily activities of the residents with the highest public level. nodeB is the worship space of the settlement and its value is highest (733) and latest in saturation (12steps). For residents, it is an important and private space. However, it is socially and culturally meaningful to the settlement. It is relatively peripherals in terms of behavior and activities.

The center and edge is a relative relationship, and an effective concept to analyse the spatial structure. In the basic mathematical topological study, the summary and analysis of the numerical computation and field investigation results suggest that the "settlement centre/edge" of the settlement spatial structure can be analysed through parameters. As the space defined in this study includes the virtual and physical space, it covers all the possible activities in the settlement relating to the behaviours of residents, the perceptions of people and the exchange of people. Therefore, the so-called centre is the location at the hub of the settlement; by comparison, the edge in this study refers to the edge of the daily life activities.

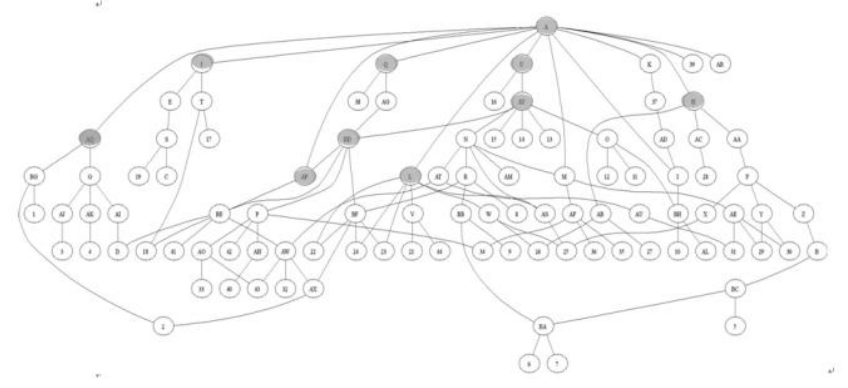

Fig. 5 The first $20 \%$ saturated nodes

(2) Key gateway concept: the space reaching saturation in the second place including the each area's key gateway Fig.5. 
From the key gateway, we can learn the social relations of the areas where different residential groups live. The concept of Gateway likes Kevin Lynch's "Node” [6].

The first $20 \%$ saturated nodes are keys of partitions. There are 11 nodes. nodeA is the most important route entering the settlement, and thus it is most open and public in the space. node $\mathrm{U}$ (356), node L(357), node $\mathrm{J}(360)$, node AQ(370), node $\mathrm{H}(371)$, node $\mathrm{AP}(374)$, node $\mathrm{Q}(378)$, node $\mathrm{AY}(378)$, node $\mathrm{BD}(386)$ are the gateways or blank spaces connecting various secondary important building complex in the settlement. Its numerical features are consistent with the records of field study, indicating that the proposed method can be used to find the key gateways of the settlement. In addition to the implications of the key nodes, these nodes are places of concentration of residents. These nodes have opportunities to form clusters in spatial perception, that is, a gateway is close to the concept of "life circle" of collective life.

(3) Inaccessibility can be indicated by the later saturated nodes. There are 11 nodes with the latest $20 \%$ depth values. These nodes represent the places that are not easily accessible in the settlement. The node of the highest depth value is node B (733) representing the hall of the settlement (Shenji Hall). It is the most important worship and ritual space in the settlement. The accessibility in daily life is the lowest; however, it is relatively important in cultural dimension.node AS (597), nodeBA(621), nodeAT(526), nodeBB(540), node nodeF $(525), \mathrm{BC}(516)$ are in the stage of latest $20 \%$ in terms of depth value with more steps, representing the space of inner courtyard or peripheries of the settlement. During the interview process, it is confirmed most residents seldom come to such space while the frequency of daily activities of most residents is not very high.
The traditional settlement space that gradually evolves from each resident's living pattern is closely related to the geographical characteristics, social and cultural features of the space. Therefore, each settlement has its own unique, hidden social spatial culture.

This study applied the topology theory in the study of the relationship between settlement space and social relations. By describing the spatial relationship in a logic way, the method for describing the relationship between the abstract spatial structure and activities is deduced. When facing the complex settlement implications, a systematic analysis is provided to understand the spatial logic and spatial structural relationship in traditional settlement. It can also serve as a reference for the community planning in the future from the cultural and social perspectives to create a sense of local culture or reproduce a sense of site planning.

\section{References}

[1] Hillier, B., Hanson, J., the Social Logic of Space. Cambridge, Cambridge University Press, 1984.

[2] Li, Chi-Hua. Lin, Feng-Tyan, A Space Syntax Analysis of Settlements in Taiwan, Journal of Architecture, No.60, pp 27 45, Jun. 2007

[3] Li, Chi-Hua. Lin, Feng-Tyan, Exploring Spatial Structures of Settlements by Saturation, Annals of GIS, pp.55-66, 2009.

[4] A Study of Topologically Culture Space on Taiwanese Settlements. Doctoral Dissertation of Building and Planning, National Taiwan University, 2010.

[5] Lin,F.T., http://www.bp.ntu.edu.tw/phpbb/viewtopic.php?p=1514\&sid=896595d 84e24a2fc8954a31c0cfc2b53, 2001.

[6] Lynch, Kevin. The Image of The City. Cambridge, Mass.: MIT Press, 1960.

[7] Mitchell, William J., Computer-aided architectural design. New York: Van Nostrand Reinhold, 1997.

[8] Mitchell, William J., The logic of architecture Design, computation, and cognition. Cambridge, Mass.: MIT Press, 1989. 